\title{
GALILEO SPACECRAFT VIEWS OF EUROPA
}

\author{
R. GREELEY \\ Arizona State University \\ Department of Geology \\ Box 871404 \\ Tempe, AZ 85287-1404 \\ AND \\ THE GALILEO IMAGING TEAM
}

\begin{abstract}
The Galileo orbiter and its Solid State Imaging system have increased the photographic coverage and resolution of Europa, revealing a complex surface that has been modified by tectonic deformation, impact craters on a wide range of scales, and possible cryovolcanic activity. The morphology of many of the features suggests that the depth to either liquid water or mobile-ductile ice was relatively shallow ( $<$ few kilometers) at the time of their formation. Characteristics of the mottled terrain and impact ejecta deposits suggest the presence of a shallow $(\sim \mathrm{km})$ subsurface zone beneath the bright icy crust. The Galileo Europa Mission, approved to continue orbital operations through 1999, will include at least nine close flybys of Europa, enabling global and regional mapping, along with high-resolution imaging of selected areas.
\end{abstract}

\section{Introduction}

Europa is about the size and density of Earth's moon. Modeling and geophysical measurements obtained by the Galileo spacecraft suggest that it has a metallic core surrounded by a rocky mantle and an outer shell of water 100 to $200 \mathrm{~km}$ thick, part of which could be liquid (Schubert et al., 1986; A rderson et al., 1997). Although Voyager images are limited in coverage and resolution (best images are about $2 \mathrm{~km} /$ pixel; Smith et al., 1979a,b), they allowed initial mapping of the surface and geological characterization of Europa (Lucchitta and Soderblom, 1982; Malin and Pieri, 1986). The surface of the satellite is criss-crossed with dark linear features set in a background of bright icy plains and mottled terrain. The general paucity of superposed impact craters led to the conclusion that the surface of Europa is geologically young, and that it could experience active resurfacing driven by tidal heating (Cassen et al., 1979).

The Galileo orbiter around Jupiter began returning new data for Europa in July 1996 and is scheduled to take images on 9 of the 11 orbits in the nominal mission. Three of the orbits include close flybys of Europa, returning images with resolutions as high as $\sim 21 \mathrm{~m} /$ pixel. Preliminary analyses of the images (Belton et al., 1996; Carr et al., 1997; Greeley et al., 1997) are providing new insight into this unique satellite.

The surface of Europa (Fig. 1) includes bright plains, mottled terrain, small mountains as high as $2 \mathrm{~km}$, impact structures, and a variety of ridges ranging in size from the limits of resolution (10s of $\mathrm{m}$ ) to $10 \mathrm{~km}$ wide by $>1000 \mathrm{~km}$ long. In many areas, the surface has been further modified by faulting, fracturing, upwarping, and degradation by an unknown process. The style and variety of features indicate complex geology, the history of which is only beginning to emerge.

\section{Geological Features}

Bright plains form broad expanses of ice which locally contain irregularly shaped depressions (pits), dark spots (leniculae), and plateau-like features less than a few hundred meters high and smaller than 10-16 km across. False-color Galileo images show in the infrared (IR) that the bright plains 


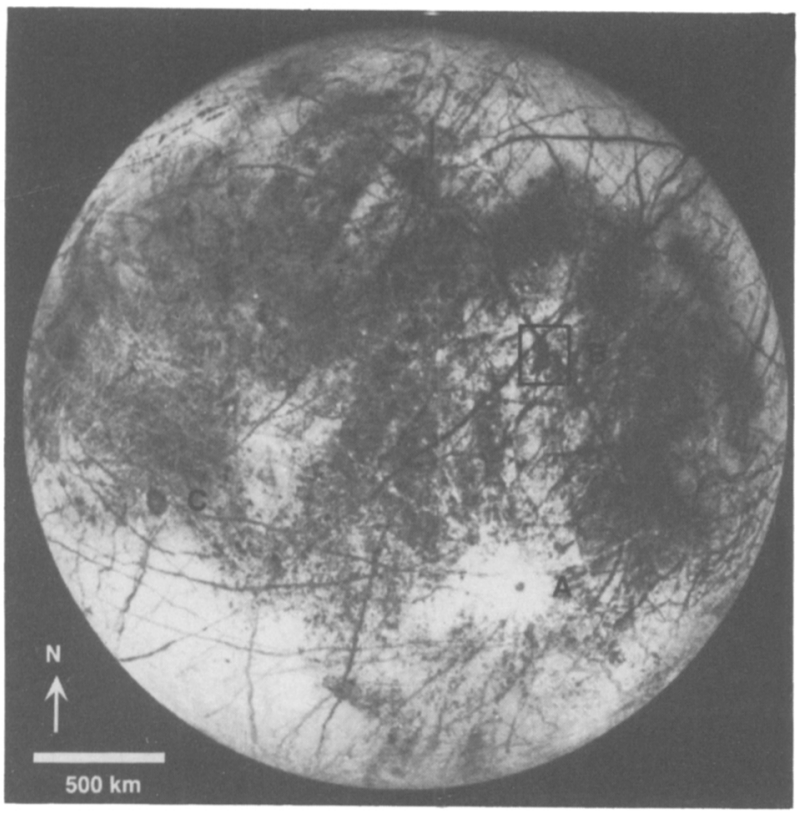

Figure 1. Galileo view of Europa centered at $285^{\circ}$ longitude, showing bright plains (lower left), mottled terrain (dark splotches), and various lineae. " $A$ " is Pwyll Crater and its bright ejecta deposits; "B" is an area named Conamara Chaos and outlines the area shown in Fig. 2; "C" is the circular impact scar named Callanish (Galileo frame S0360063913).

subdivide into IR-dark and IR-light units, which could be related to differences in ice-grain sizes or perhaps composition (Belton et al., 1996).

Mottled terrain covers large parts of Europa and consists of irregular dark splotches 10s of kilometers or more across. Mottled terrain is particularly dominant in the equatorial region while only a few patches occur in the polar areas. This terrain appears to form at the expense of the bright plains (and, hence, is younger) by the breakup of the icy crust to expose an underlying unit containing non-ice components (Fig. 2). Near Infrared Mapping Spectrometer data (Carlson et al., 1997) suggest that this component includes hydrated silicates such as clay minerals.

Impact craters range in size from the limits of detection ( hundred meters in diameter) to Pwyll, a 26-km diameter bright-rayed crater. Pwyll exhibits low albedo ejecta deposits that can be traced some $12 \mathrm{~km}$ from the crater rim. Some impact craters a few kilometers across also display dark ejecta and rays. These observations suggest that a zone of dark material exists less than a kilometer beneath the ice-rich surface, at least in some areas of Europa.

Some large dark spots, or maculae, are also probably impact scars. Callanish is a circular feature defined by grabens and other fractures forming a concentric ring more than $100 \mathrm{~km}$ across; secondary-like craters occur in patches outward from the ring fractures. Tyre Macula is a similar appearing structure 120 by $160 \mathrm{~km}$ across. Both of these features could reflect impacts into the icy crust which either relaxed by long-term viscous flow, or which formed only a transient cavity at the time of impact without the preservation of a craterform.

Impact crater size-frequency distributions have been obtained for Europa, Ganymede, and Callisto, and compared with other cratered objects in the Solar System (Neukum et al., 1997). However, debate has arisen with regard to the ages of Europan surfaces based on the crater frequencies, depending on the method of calibration. Shoemaker (1996) suggested very young surfaces - perhaps $3 \times 10^{6}$ years or less - while Neukum et al. (1997) extrapolate from calibrated lunar data and adjust for consistency among the Jovian satellites, and suggest ages greater than $3 \times 10^{9}$ years.

Some areas of Europa seen in high resolution $(21 \mathrm{~m} / \mathrm{pixel})$ appear resurfaced, either by extrusion of material onto the surface or mobilization of surface ice by melting. Other areas seen in lower 


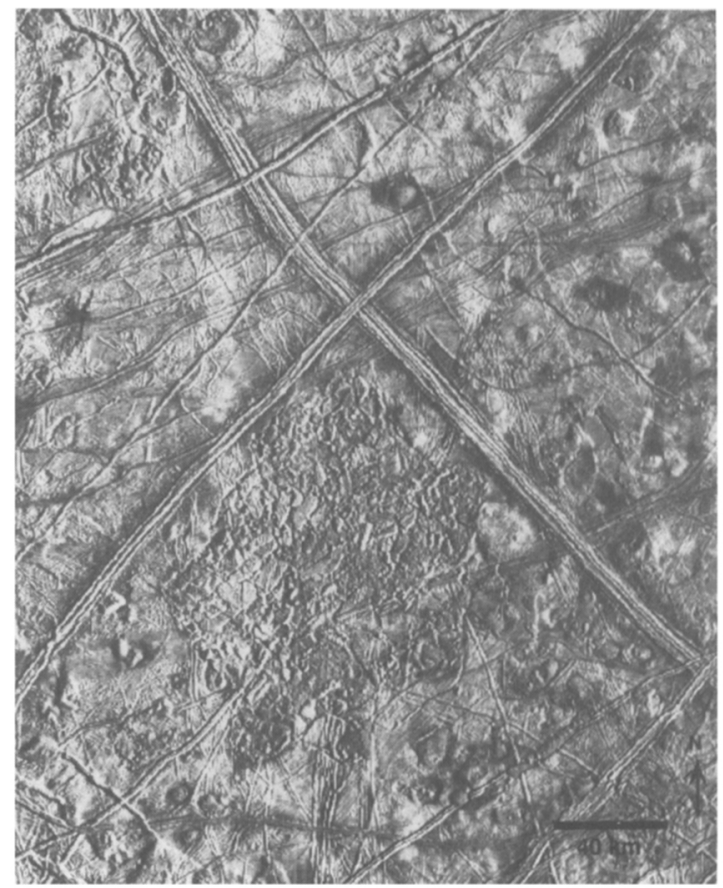

Figure 2. Galileo image showing the intersection of Asterius Linea (lower left to upper right) and Agave Linea (upper left to lower right). Conamara Chaos is the disrupted terrain south of the intersection and is shown in more detail in Fig. 4. Several dark, dome-shaped features, termed lenticula, are also visible. Illumination is from the left. Image has a resolution of $180 \mathrm{~m} /$ pixel and is courtesy of Cynthia Phillips, University of Arizona.

resolution $(630 \mathrm{~m} /$ pixel $)$ show glacial flow-like features more than $16 \mathrm{~km}$ across which appear to have cut through, or breached older ridges.

Some lenticulae appear to be areas that have been upwarped from below (Pappalardo et al., 1997). Typically, these areas are less than $10 \mathrm{~km}$ across, many of which are surrounded by shallow moats. The surface of the upwarp is commonly fractured like a bread crust or disrupted into a chaotic terrain. Upwarping could result from convection (a local "hot spot"), intrusion of ductile ice, or diapiric uprising of lower density materials (Greeley et al., 1997).

Lineae were defined from Voyager images for any of a variety of band-like features, including high and low albedo stripes, ridges, and triple bands consisting of parallel low albedo stripes separated by a high albedo zone. Galileo images show that many lineae are complex ridges and grooves. Most investigators consider lineae to result from tectonic processes in which the ice crust is fractured, followed by extrusions of ductile ice or slush, and/or compression of ice plates; there might also be explosive venting from the interior to produce geysers along the fractures (Greeley et al,, 1997). Although mapping the global patterns of various lineae is not complete (awaiting additional images), initial results suggest that the Europan crust has been fractured in response to non-synchronous rotation of the satellite around Jupiter (Greenberg et al., 1997; Geissler et al., 1997).

Various wedge-shaped bands $>100 \mathrm{~km}$ long were also described previously. A particularly prominent set occurs near the anti-Jovian region and was imaged by Galileo at about $420 \mathrm{~m} / \mathrm{pixel}$ (Fig. 3; Sullivan et al., 1997; Tufts et al., 1997). These images show that some wedge-shaped bands are places where the crust has been separated with the intervening area filled with lower albedo materials, somewhat analogous to sea-floor spreading centers on Earth. However, the area on Europa has been disrupted much more intensely, leading to the term "pull-apart" terrain.

The crust of Europa has also been disrupted locally, giving the appearance of icebergs set in a frozen matrix. One such area, shown in Fig. 4 and named Conamara Chaos, includes ridged and grooved terrain that has been broken into plates which have been repositioned and in some cases 


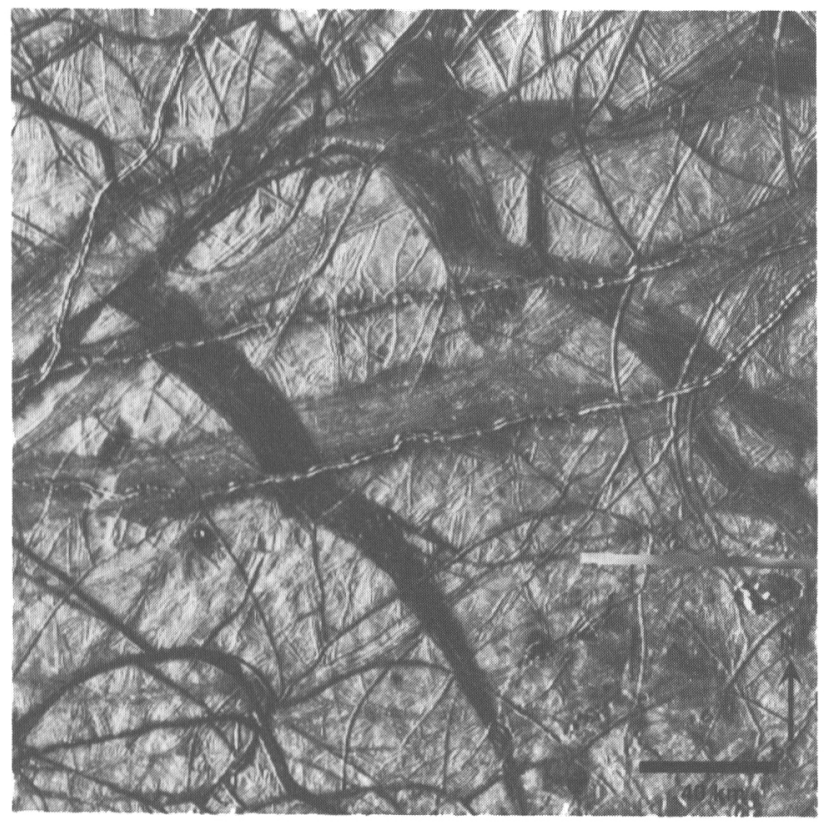

Figure 3. Moderate resolution ( $460 \mathrm{~m} / \mathrm{pixel})$ view of the "pull-apart" terrain showing dark wedge-shaped bands, triple bands, and the complex background matrix of complex ridged and grooved terrain. Also visible is a small impact crater with dark ejecta deposits (middle-left). Illumination is from the left. (Galileo JPL P-48127).

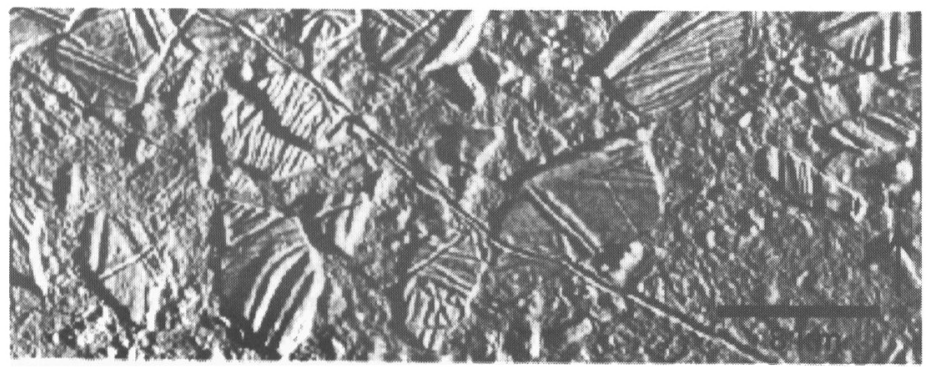

Figure 4. High resolution ( $54 \mathrm{~m} / \mathrm{pixel}$ ) Galileo view of part of Conamara Chaos near $9.5^{\circ} \mathrm{N}, 273^{\circ} \mathrm{W}$ showing "plates" of ridged and grooved terrain set in a matrix of chaotic terrain. Many of the plates can be fit together like pieces of a jigsaw puzzle. Illumination is from the right (Galileo JPL P-48526).

tilted on edge as though they are being submerged into the matrix. The matrix has the appearance of having been very low viscosity at the time of disruption, suggesting liquid water or water-rich material at or very near the surface. Alternatively, the matrix could have been ductile ice. In either event, there must have been sufficient energy, i.e., heat, close to the surface to have disrupted the terrain to its present morphology.

\section{Summary}

Thus far in the Galileo mission, imaging has been primarily reconnaissance and exploratory, rather than systematic mapping. Consequently, we are not yet in a position to generalize the evolution of Europa's surface. From the initial results, however, some factors are beginning to emerge. Parts of Europa have clearly been subjected to intense surface disruption, suggesting near-surface heat sufficient to mobilize the ice crust, perhaps to the melting point, or to have erupted low viscosity materials onto the surface. It is not known if these processes occurred globally, nor if they are taking 
place today. The Galileo Europa Mission, currently planned to continue through December, 1999, will involve repeated flybys of Europa, increasing both the global mapping and high resolution imaging of selected regions.

\section{References}

Anderson, J.D. et al. (1997) Science, 276, pp. 1236-1239.

Belton, M.J.S. et al (1996) Science, 274, pp. 377-385.

Carlson, R. et al. (1997) In: this volume.

Carr, M.H. et al. (1997) Nature, (submitted).

Cassen, P.M. et al. (1979) Geophys. Res. Lett., 6, pp. 731-734.

Geissler, P.E. et al. (1997) Nature, (submitted).

Greeley, R. et al. (1997) Icarus, (submitted).

Greenberg, R. et al. (1997) Icarus, (submitted).

Licchitta, B.K. and L.A. Soderblom (1982) In: Satellites of Jupiter, D. Morrison, ed, University of Arizona Press, Tucson, pp. 521-555.

Malin, M.C. and D.C. Pieri (1986) Europa. In: Satellites, J.A. Burns and M.S. Matthews, eds., University of Arizonit Press, Tucson, pp. 689-716.

Neukum, G. et al. (1997) In preparation.

Pappalardo, R.T. et al. (1997) Nature, (submitted).

Schubert, G. et al. (1986) In: Satellites, J.A. Burns and M.S. Matthews, eds., University of Arizona Press, Tucson, pp. 224-292.

Shoemaker, E.M. (1996) In: Europa Ocean Conference, San Juan Capistrano, November 12-14, pp. 65-66.

Smith, B.A. et al. (1979a) Science, 204, pp. 951-972.

Smith, B.A. et al. (1979b) Science, 206, pp. 927-950.

Sullivan, R. et al. (1997) Lunar Planet. Sci. Conference, 28, Abstracts, pp. 1395-1396.

Tufts, B.R. et al. (1997) Lunar Planet. Sci. Conference, 28, Abstracts, pp. 1455-1456. 